\title{
Innovative aperture segmentations control image plane diffraction
}

James Breckinridge, Tony Hull, James Harvey

James B. Breckinridge, Tony Hull, James E. Harvey, "Innovative aperture segmentations control image plane diffraction," Proc. SPIE 11852,

International Conference on Space Optics - ICSO 2020, 118524Z (11 June 2021); doi: 10.1117/12.2599823 SPIE. Event: International Conference on Space Optics - ICSO 2021, 2021, Online 


\section{International Conference on Space Optics-ICSO 2020}

Virtual Conference

30 March-2 April 2021

Edited by Bruno Cugny, Zoran Sodnik, and Nikos Karafolas
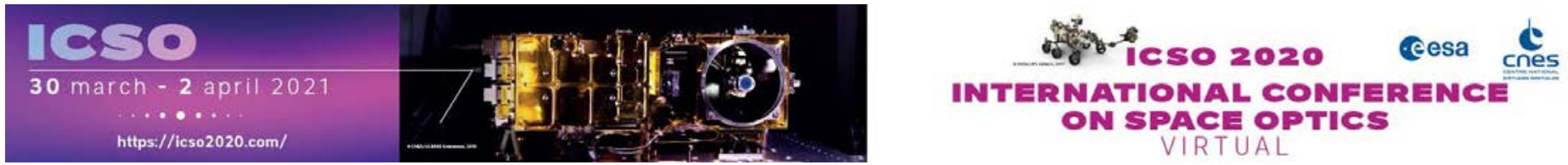

\section{Innovative aperture segmentations control image plane diffraction}

\section{Cesa isopmeatians ecnes}

International Conference on Space Optics - ICSO 2020, edited by Bruno Cugny, Zoran Sodnik, Nikos Karafolas, Proc. of SPIE Vol. 11852, 118524Z - (c) 2021 ESA and CNES

CCC code: $0277-786 \mathrm{X} / 21 / \$ 21 \cdot$ doi: $10.1117 / 12.2599823$ 


\title{
Innovative aperture segmentation controls image plane diffraction
}

\author{
James B. Breckinridge ${ }^{1,2}$, Tony Hull ${ }^{3}$, James E. Harvey ${ }^{4}$
}

1. College of Optical Sciences, U of A, 2. Astronomy Department, Caltech, 3. University of New Mexico, Albuquerque, NM. 4 Photon engineering, LLC, 310 S Williams Blvd \#222. Tucson, AZ 857114.

\begin{abstract}
Several modern astrophysics and exoplanet science imaging problems require minimum diffraction across the image plane. All large aperture ground and space telescopes today use a hexagonal segment pattern to tile the aperture. These tiles are optically phased to synthesize a single large aperture. This hexagonal pattern produces fixed pattern noise across the image which masks important astrophysics and exoplanet details. We present a new segment topology we call the pinwheel which eliminates this fixed pattern noise while enabling a cost effective aperture fabrication process. This new process lend itself to replication techniques which may reduce the aperture cost per unit area to that below the hex pattern topology. We evaluate this new topology for applications in exoplanet characterization science.
\end{abstract}

\section{INTRODUCTION}

In this paper we review technologies to minimize the diffraction spatial noise introduced by implementing hexagonal or straight-line shaped segments to tile large aperture space telescope mirrors. In addition we describe a way forward to develop processing technology that will enable the innovatively shaped segments to minimize segment edge noise.

\subsection{Linear systems theory for image formation}

\section{BACKGROUND}

Object space irradiance distribution can be decomposed into an ensemble of delta functions. The intensity or height of each delta function maps out the structure of the object. The optical system operates on the complex amplitude and phase associated with that intensity distribution to form an image at the detector. Most astronomical sources in the visible region of the spectrum radiate broadband, incoherent thermal light.

The theory of image formation is developed using the schematic shown in Figure 1 below. The coordinate system we use in our analysis is shown in Fig 2 below. This system is in standard use by modern textbooks ${ }^{1},{ }^{2}$ on the physics of image formation. Light travels left to right. The object plane to the left (\#1 in the system) is represented by Cartesian coordinates from the Latin alphabet $x_{1}, y_{1}$ the pupil plane (\#2 in the System) is represented by Cartesian coordinates from the Greek alphabet $\xi_{2}, \eta_{2}$ and the image plane (\#3 in the System) is represented by Cartesian coordinates from the Latin alphabet $x_{3}, y_{3}$.

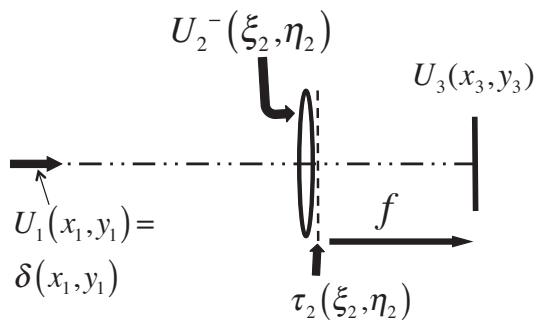

Figure 1 schematic of an optical system in the meridional plane. The object space complex field $U_{1}\left(x_{1}, y_{1}\right)$ is shown as a delta function $\delta\left(x_{1}, y_{1}\right)$ to represent a star on- 
axis. This object space field is propagated by Fresnel diffraction to just to the left of the entrance pupil and is shown by $U_{2}^{-}\left(\xi_{2}, \eta_{2}\right)$. The pupil is shown to have a complex transmittance $\tau_{2}\left(\xi_{2}, \eta_{2}\right)$ at plane 2. The optical system, shown here schematically has a lens of focal length $f$, and provides the optical power to create the field $U_{3}\left(x_{3}, y_{3}\right)$ at an image plane 3.

The scalar complex amplitude and phase across the image plane is found by standing at the image plane (\#3) in Fig 2 and looking to the left, or back through the system toward the object. The Fresnel-Kirchoff diffraction integral is used to model the propagation of scalar electromagnetic waves through the optical system shown in Fig. 1. The complex amplitude and phase field $U_{3}\left(x_{3}, y_{3}\right)$ at the image plane is given by

$$
\begin{aligned}
& U_{3}\left(x_{3}, y_{3}\right)= \\
& \mathrm{K} \int_{-\infty}^{\infty} \int_{-\infty}^{\infty}\left[U_{2}^{-}\left(\xi_{2}, \eta_{2}\right)\right] \cdot \tau_{2}\left(\xi_{2}, \eta_{2}\right) \cdot \exp \left\{-j \frac{2 \pi}{\bar{\lambda} f}\left(x_{3} \xi_{2}+y_{3} \eta_{2}\right)\right\} d \xi d \eta
\end{aligned}
$$

Where $\mathrm{K}$ is a constant, the integral is taken over the complex field across the exit pupil, $U_{2}^{-}\left(\xi_{2}, \eta_{2}\right)$ of the optical system whose focal length is $f, \bar{\lambda}$ is the quasimonochromatic wavelength of light. Eq 1 is written for the scalar wave solution to Maxwell's equations and the not vector wave solution to Maxwell's equation. The amplitude and phase complex properties across the exit pupil are contained in the scalar term,

$$
\tau_{2}\left(\xi_{2}, \eta_{2}\right)=A_{2}\left(\xi_{2}, \eta_{2}\right) \exp \left\{i \phi_{2}\left(\xi_{2}, \eta_{2}\right)\right\}
$$

Where $A_{2}\left(\xi_{2}, \eta_{2}\right)$ varies between 0 and 1 and describes amplitude part of the complex wave as a function of position across the exit pupil. The phase properties at each point across the exit pupil are described by $\phi_{2}\left(\xi_{2}, \eta_{2}\right)$.

To the left in Fig 1, we have a point source represented by a delta function. This point source is mapped onto the image plane. We record intensity at the image plane and define the image plane irradiance distribution for this point source to be:

$$
P S F_{\text {Scalar Diffraction }} \equiv\left|U_{3}\left(x_{3}, y_{3}\right)\right|^{2} \text {. }
$$

Next if we let the object space irradiance be represented by $I_{\text {Object }}\left(x_{1}, y_{1}\right)$ and the image space irradiance represented by $I_{\text {Image }}\left(x_{3}, y_{3}\right)$ and use the theoretical development of Goodman, we can write,

$$
I_{\text {Image }}\left(x_{3}, y_{3}\right)=I_{\text {Object }}\left(x_{1}, y_{1}\right) \otimes P S F,
$$

Where the symbol $\otimes$ denotes the convolution operator.

\subsection{Static and dynamic components of the PSF}

The complex amplitude and phase field $U_{3}\left(x_{3}, y_{3}\right)$ at the image plane given by eq 1 can be decomposed into linear terms representing dynamic changes to the optical system and those static terms given by the optical systems engineering terms: geometric wavefront aberrations, polarization aberrations, scattered light and diffraction. Dynamic changes occur during integration time and changes to the system that results from repointing the telescope/instrument system. Dynamic changes to the optical system are produced by telescope and instrument 
pointing and tracking errors along with changes to the optical system introduced by timedependent thermal gradients which change the spacing of optical elements and their precision alignment.

The optimum STATIC point spread function (PSF) for an optical system that is designed for threshold detection is one that exhibits both minimum PSF shape distortion ${ }^{3}$ and minimum spatial structure ${ }^{4}$. Several end-to-end optical system factors interact to affect the PSF shape distortion and its spatial structure. These are:

1. Geometric wavefront aberrations which are understood using trigonometry and controlled using innovative opto-mechanical layouts and adaptive optics (A/O),

2. Polarization aberrations which are understood using vector wave theory and controlled using innovative opto-mechanical layouts, optical coatings and spatially variable wave plates ${ }^{5}$,

3. Diffraction which is controlled by adjusting the shape of shadows and segment gaps across the end-to-end optical system exit pupil are understood using scalar \& vector wave theory, and

4. Scattered light which is understood using scalar and vector wave scattering models and controlled using innovative opto-mechanical layouts, highly transparent and homogeneous optical windows, creative surface polishing technologies and coatings.

Geometric wavefront aberrations are introduced by mirror-surface fabrication errors. Polarization aberrations arise from the dielectric and metal coatings on mirror surfaces needed to maintain a high optical-transmittance system. Surface scatter arises because of limitations to our ability to fabricate perfectly smooth surfaces. Diffraction arises in Cassegrain telescope systems from shadows cast on the primary mirror by the mechanical structure needed to support the secondary mirror. Diffraction is also introduced by the gaps between primary-mirror segments needed to create a large aperture telescope mirror which must be deployed or assembled in space because of limited lift mass and volume capacity for modern launch vehicles.

The remainder of this paper concentrates on innovative space telescope architectures which mitigate the deleterious effects of diffraction on image quality for threshold astrophysics, earth, and planetary space science. We select one particular observational requirement, that of direct imaging of terrestrial exoplanets at the threshold level to examine further.

\section{SEGMENT TOPOLOGY}

Direct imaging of terrestrial exoplanets is necessary if astronomers are to obtain detailed spectra of a planet's surface and atmosphere to characterize planetary evolutionary tracks and estimate the probability that the planet is capable of supporting life. The apparent angular separation between the exoplanet and its parent star is less than one-arc second. Exoplanets are much smaller in size than the parent star and shine in light reflected from that star. The ratio of light from the planet to that of the star is between $10^{-10}$ and $10^{-11}$, depending on the size of the planet and its separation.

Space telescope apertures of 15 to 30 meters are needed to provide the angular resolution and the radiation-gathering power to produce a significant statistical sample of terrestrial exoplanets for analysis. A telescope aperture of this size cannot be placed in orbit fully erected, rather mirror segments are either folded into a smaller volume (as was done for JWST) or in the future may be assembled in space ${ }^{6}$. Therefore, space telescopes of 15 to 30 -meter aperture will 
be partitioned into individual segments. To allow for deployment or assembly in space, the telescope aperture is divided up into separate mirrors with gaps between mirrors to produce a discontinuous concave surface. These gaps diffract light across the image plane. If these gaps form periodic linear structures across the aperture, then this diffracted light produces structured, unwanted radiation across the image to mask exoplanets.

The standard space telescope architecture today is that of a Cassegrain telescope, which has a secondary mirror supported by 4 or 3 structural beams that shadow the telescope aperture, as well as a hole on the primary mirror to allow light reflected from the secondary to pass thought and into a science instrument module. The NASA next generation large telescope architecture: Large UV Optical IR (LUVOIR) uses this Cassegrain telescope architecture, along with a primary mirror that is divided into regular hexagonal shaped segments. In this case, the telescope entrance pupil is discontinuous because of both the segment gaps and the secondary support structure shadows.

In this paper, combined with the following paper ${ }^{7}$ we show that by using curved structures for the secondary support system and curved sides to nest the segments one adjacent to the other across the primary mirror, we nearly eliminate the image plane "diffraction-noise" by disrupting the diffraction pattern and creating a nearly uniform background across the image plane.

The advantages of implementing this architecture are: 1 . Eliminate the need for exotic and absorbing apodizing masks which control diffraction from segment gaps, 2. Increase exoplanet characterization data quality, 3 . Improve radiometric calibration, 4. More accurate image restoration, since the point spread function (PSF) is both rotationally symmetric and isoplanatic over a FOV.

\section{WHERE ARE THE TERRESTRIAL EXOPLANETS?}

To identify terrestrial exoplanet in the FOV we calculate the FOV location of an Earth twin at the distance of the parent star. We estimate its's detectability by calculating the telescope aperture in meters needed to place the Earth twin at the first and third ring of the Airy diffraction pattern at the image plane. The contents of table 1 identifies the location of terrestrial exoplanets and shows telescope apertures needed to record them. Note we assume the exoplanet is at elongation in its orbit, a position the planet occupies only a short time. This makes it very important that we know the orbital details of exoplanet systems we observe to maximize our probability of getting good spectra.

Table 1 Angular separation of an Earth-twin terrestrial-exoplanet from its solar-like parent at elongation as a function of distance from the solar system in units of parsecs is shown. Column 1 gives the distance from our solar system to the candidate exoplanet system. Column 2 shows the apparent angular separation between the primary star and its terrestrial exoplanet at elongation in its orbit. Column 3 presents the telescope aperture needed to just resolve this separation. Column 4 gives the telescope aperture needed to place the exoplanet at the third diffraction ring of the clear aperture. In both cases the telescope apertures are calculated for $500-\mathrm{nm}$ wavelength. 


\begin{tabular}{|c|c|c|c|}
\hline $\begin{array}{c}\text { Distance } \\
\text { Parsecs } \\
\text { PC }\end{array}$ & $\begin{array}{c}\text { Angle } \\
\text { between star } \\
\text { \& Earth twin } \\
\text { in milli-arc - } \\
\text { sec }\end{array}$ & $\begin{array}{c}\text { Aperture in } \\
\text { meters } \\
\text { Diffraction } \\
\text { limited at 500nm }\end{array}$ & $\begin{array}{c}\text { Aperture in } \\
\text { meters third } \\
\text { Airy diffraction } \\
\text { ring }\end{array}$ \\
\hline 10 & 100.0 & 1.2 & 3.7 \\
\hline 20 & 50.0 & 2.5 & 7.5 \\
\hline 30 & 33.3 & 3.7 & 11.1 \\
\hline 40 & 25.0 & 5.0 & 15.0 \\
\hline 50 & 20.0 & 6.2 & 18.6 \\
\hline 60 & 16.7 & 7.4 & 22.2 \\
\hline 70 & 14.3 & 8.7 & 26.1 \\
\hline 80 & 12.5 & 9.9 & 29.7 \\
\hline 90 & 11.1 & 11.1 & 33.3 \\
\hline 100 & 10.0 & 12.0 & 36.0 \\
\hline
\end{tabular}

The Hipparcos catalog shows that there are 2347 stars with measured parallaxes of $\mathrm{pi}=$ 33.33 mas, which correspond to a distance of $30 \mathrm{pc}$ down to stellar magnitude $\mathrm{V}=8^{8}$. Exoplanets are $\sim 10^{10}$ fainter than their parent star. If the parent star has magnitude $\mathrm{V}=8$, then the faintest terrestrial exoplanets within $30 \mathrm{pc}$ will be between stellar magnitudes 31 and 34. For reference, the Hubble (2.4-m) ultra-deep field magnitude limit is $\sim 29$ and required an exposure of approximately $2 * 10^{+5}$ seconds 9 .

\section{PHYSICAL OPTICS OF DIFFRACTION}

Discontinuous telescope mirrors, that is telescope apertures that have some portion of the aperture blocked are responsible for diffraction "noise" at the image plane. Breckinridge, Kuper and Shack (1982) ${ }^{10}$ were the first to discuss the role of secondary support diffraction spikes in finding exoplanets. Figure 1 below shows the diffraction pattern from the HST caused by the secondary support structure. Near the star we also see the "diffuse-light" effects of narrow angle scattered light. The sources of narrow angle scattered light in telescope/coronagraph systems were discussed by Pfister, et. al. ${ }^{11}$

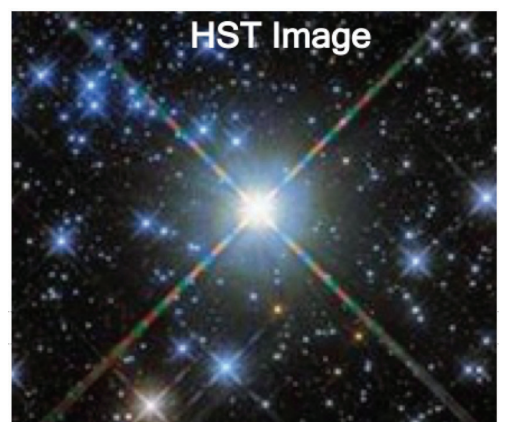

Figure 2 HST image of a star showing the diffraction spikes that mask exoplanets at 4 position angles. The halo around the star is produced in the telescope/instrument system by narrow angle scattered light. The Airy diffraction pattern for HST is about $\mathbf{1 0 0}$ milliarc seconds which is too large to observe terrestrial exoplanets 1 astronomical unit from the parent star. 


\section{Segmented space telescope diffraction}

Today, space-telescopes that are segmented use close-packed regular hexagon-sided mirrors to package their reflective surfaces into a nearly circular telescope pupil. Figure 4 below shows, on the left, one of the concept apertures for the Large Ultra-Violet Infrared (LUVOIR) mission study. On the right we see the individual segments of the primary. This telescope primary exhibits three diffraction gratings which diffract light from the much brighter central parent star across the image plane and create background noise for imaging and spectroscopy of exoplanets. The gaps between segments appear as discontinuous diffraction grating rulings across the aperture The direction of the "rulings" of these three diffraction gratings are shown using red, blue and orange arrows in Figure 4.

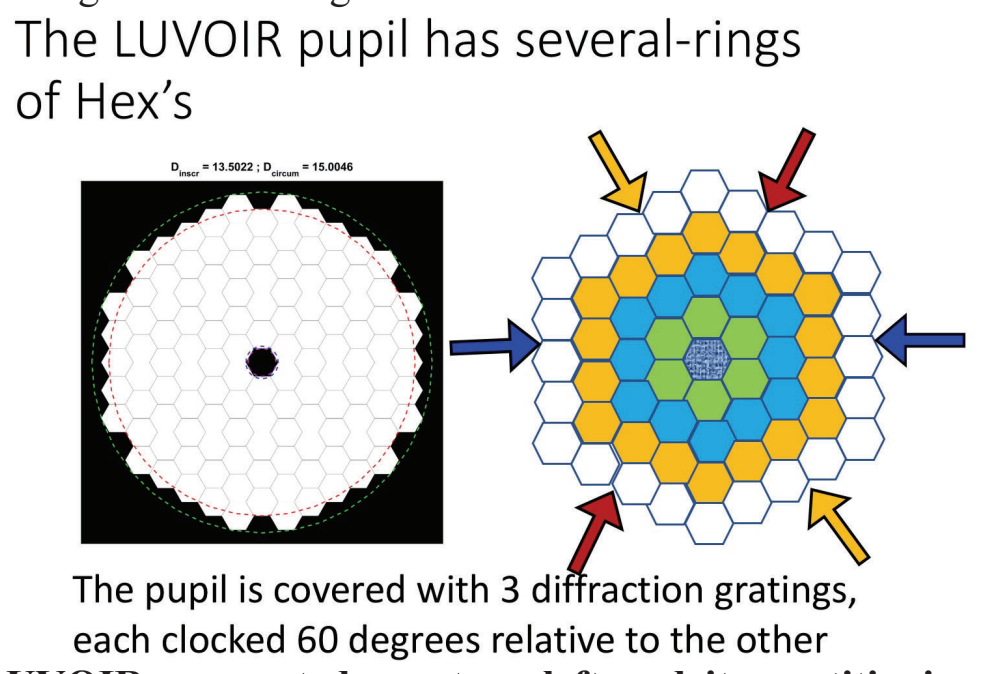

Figure 3 LUVOIR segmented aperture left and its partitioning into three linear diffraction gratings. The 3 "ruling" directions are clocked 60-degrees in relation to each other. The directions of the grating rulings are shown by the arrows in the three colors: red, blue and orange.

The rulings are discontinuous across the LUVOIR pupil. Equation 1 has been interpreted to show that there is a spatial frequency Fourier Transform relationship between the complex field across the telescope aperture and the image plane ${ }^{1}$. Therefore, the diffraction grating rulings formed by the gaps between the segments are shift invarient and the discontinuity of the gap lines (grating rulings) are shift invariant and result in a decrease in intensity of the diffraction orders but does not alter their presence. Figure 4 shows 2 of the 3 sets of diffraction grating rulings across the hexagonally segmented LUVOIR pupil. The third set of rulings, the horizontal set are not shown to avoid confusion in the drawing. 


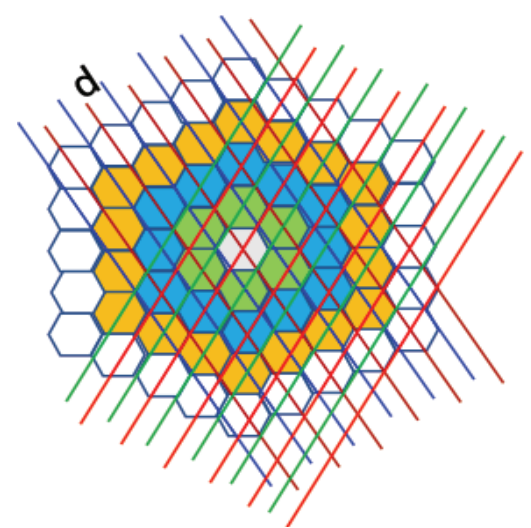

Figure 4 Two of the three diffraction gratings across the close-packed hexagon-segmented primary mirror are shown. The spacing of the "ruling" is $d$ and the rulings are "clocked" or rotated 60 degrees from one set to the next.

Figure 4 shows the close-packed hexagon-segmented primary mirror with lines drawn to show the grating "rulings" and the direction of the rulings. The "groove-spacing" is seen to be d, where $\mathrm{d}$ is one-half the face-to-face distance across the individual regular hexagons. The diffraction causes a structured background across the image plane that may obscure important exoplanets and may introduce unwanted polarization aberrations into the coronagraph to affect image quality ${ }^{12}$.

\section{The PSF for a monochromatic star}

Gratings diffract light into orders which map a single on-axis point (a monochromatic star, for example) into multiple images of that monochromatic star. If the source is polychromatic then the grating maps the polychromatic single on-axis point into multiple spectral images stretched out radially. This is, of course how a diffraction grating spectrograph operates.

Let $\lambda=$ wavelength; $\mathrm{d}=$ =ruling spacing; $\mathrm{n}=$ diffraction order and $\theta=$ angle from the axis, then the grating equation that relates these 4 variables is written:

$$
n \lambda=2 d \sin (\theta)
$$

or for very small angles we can write

Equation 5.

$$
\theta=\frac{n \lambda}{2 d} \text {, where } \theta \text { is in radians, } \quad \text { Eq } 1
$$

The angular separation between diffraction orders is $\theta$ as given in Eq. 5 above.

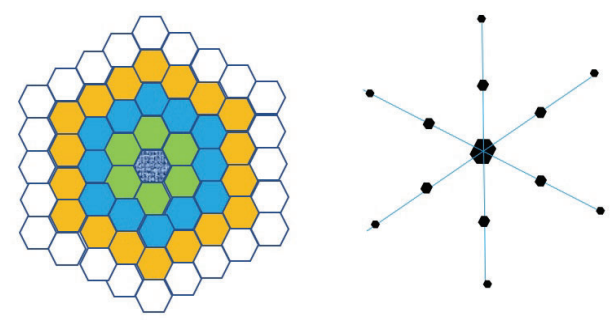

Figure 5 shows, left, a pupil map and right a representation of the monochromatic PSF associated with the close-packed hexagon-segmented primary mirror shown on the left. The points on the right-hand side show the location of the diffraction orders. The center is the image of the star and the first ring of points corresponds to the $1^{\text {st }}$ order of the three diffraction 


\section{gratings. The second ring of points corresponds to the $2^{\text {nd }}$ order of the three diffraction gratings.}

In figure 5 we see that a Lyot coronagraph occulting mask would only block light from the zero-diffraction order, which contains light from the bright central star. But light from the higher diffraction orders $\mathrm{n}>1$ will scatter around the occulting mask to flood the detector plane. The occulting mask could be designed such that each order has its own mask, but that would block portions of the FOV where exoplanets might be found.

To determine if the diffraction images of the parent star will obscure exoplanets, we calculate the angular separation between zero order and the first order, $n=1$, for polychromatic light. Table 2 below shows the angular separation of the 450, 500, $550 \mathrm{~nm}$ wavelength monochromatic diffraction orders as a function of the face-to-face segment size.

Table 2 Angular separation of the diffraction orders for face-to-face segment sizes: 1,2,3,4 meters. This applies to the diffraction orders shown in Fig. 6, above.

\begin{tabular}{|c|c|c|c|}
\hline $\begin{array}{c}\text { Face-to Face } \\
\text { segment size in } \\
\text { meters }\end{array}$ & $\begin{array}{c}\text { Angle for 450- } \\
\mathrm{nm} \\
\text { masec }\end{array}$ & $\begin{array}{c}\text { Angle for 500- } \\
\mathrm{nm} \\
\text { masec }\end{array}$ & $\begin{array}{c}\text { Angle for 550-nm } \\
\text { masec }\end{array}$ \\
\hline 1 & 93 & 103 & 113 \\
\hline 2 & 47 & 52 & 57 \\
\hline 3 & 31 & 34 & 38 \\
\hline 4 & 23 & 26 & 28 \\
\hline
\end{tabular}

We compare the entries in Table 2 with the entries in Table 1 and see that the unwanted diffraction images of the parent star fall within the same FOV region as the exoplanets. Clearly there would be a significant advantage to the development of a straightforward, low absorption way to eliminate these diffraction orders. The pinwheel pupil provides that opportunity.

\section{The PSF for a polychromatic star}

Exoplanets are very faint thermal sources. If they are to be observed in monochromatic or narrow band light integration times become impossibly long. The HabEx coronagraph is planned to observe in $100 \mathrm{~nm}$ bandwidths. One of these bandwidths in 450 to $550 \mathrm{~nm}$ and we have used those values to compute the diffraction angles shown in Table 2. The star image at $\mathrm{n}=1$ for the 1-meter face-to-face segments is a colored radial streak or small spectrum with 450 $\mathrm{nm}$ light at 93 masec and $550 \mathrm{~nm}$ light at 113 masec. The 2-meter face-to-face segments is a colored radial streak or small spectrum with $450 \mathrm{~nm}$ light at 47 masec and $550 \mathrm{~nm}$ light at 57 masec. This continues to the 4-meter face-to-face segments which give a colored radial streak or small spectrum with $450 \mathrm{~nm}$ light at 23 masec and $550 \mathrm{~nm}$ light at 28 masec.

\section{Isoplanatic point spread function for image processing}

The polychromatic PSF shown in figure 5 (right) is not linear shift invariant and therefore the optical system is not isoplanatic. Also, looking at Fig 5, right, we see that the PSF is also not rotationally symmetric. These two facts complicate digital image processing.

Below we describe a pupil segmentation or topology architecture presented in earlier papers by the authors ${ }^{13},{ }^{14},{ }^{15}$ that will produce images from an emulated filled aperture telescope pupil even though the pupil is mechanically segmented. This promises to reduce significantly the effects of an anisoplanatic PSF and will make digital image processing more reliable and less uncertain.

\section{Compensating for hexagonal segments}


Technologies to compensate for the diffraction patterns produced by straight line gaps and straight-line support structures across primary mirrors of large telescopes has been an area of active study recently $16,17,18,19,20,21$. None of these methods may be completely satisfactory, however, since light is absorbed in the process.

\section{Background}

\section{CURVED SECONDARY SUPPORT STRUCTURES NOT NEW}

Breckinridge (2018) ${ }^{22}$ suggested partitioning the primary into curved sided segments and curving the secondary support structures to reduce diffraction noise at its source to control diffraction noise at the image plane of exoplanet coronagraphs. We have shown above that the hexagonal segment architecture or pupil topology leads to unwanted diffraction noise in the system. It is good engineering practice to seek ways to eliminate or reduce "noise" at its source, rather than devise complicated and signal absorbing methods to compensate. Methods to mitigate diffraction noise were developed over the years by amateur astronomers, and later optical scientists. However, the professional space and ground astronomical optical telescope and instrument community seems not aware of these techniques.

C. H. Werenskiold (1941 $)^{23}$ reported on the work of A. Couder published in the French journal: Astronomy, Jan 1934 and translated into English and republished in Amateur Telescope Making Advanced (scientific American Publishing), pp 620-622. Couder proposed controlling diffraction in Newtonian and Cassegrain type telescopes by placing lune shaped curved masks over the straight edge support structure of the secondary. These masks blocked significantly, the light-gathering ability of the telescope, negatively affecting the telescope transmittance.

Werenskiold proposed curving the secondary support structures themselves as shown below in Fig 6 to reduce masking of the primary mirror and control the diffraction spikes.
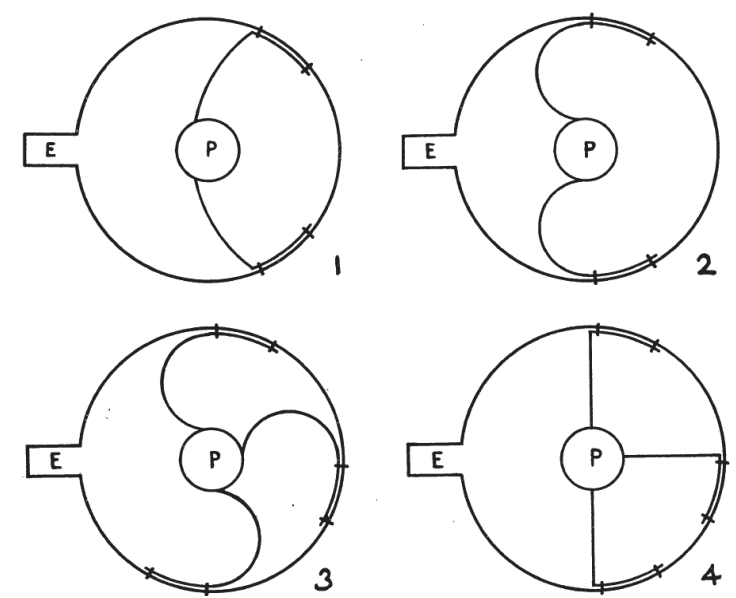

Figure 6 The four secondary support structures built by Werenskiold ${ }^{14}$ and used for visual observation of planets. View looking from the open end of a reflecting telescope back toward the primary mirror. He reports that \#4 gives the lowest quality image and that curving the support structure appears to remove the diffraction spikes from visual images to give higher contrast for visual planetary observations.

Werenskiold writes:

"It is generally conceded that a reflector, in regard to definition obtained, is apt to be somewhat inferior to a refractor of comparable 
size. However, the use of a curved spider in a reflector appears to be a promising step towards reducing this difference in comparative performance of the two telescope types."

\section{MODELLING A 10-METER PINWHEEL PUPIL}

\section{Pupil architecture of topology}

Richter ${ }^{24}$ selected 6 diffraction masks and photographed the diffraction pattern from each to show that curved arcs on the pupil left no discernible diffraction pattern at the image plane. Harvey ${ }^{25}$ (in this volume) used FRED ${ }^{26}$ and applied the design methodology outlined in Richter ${ }^{15}$ and developed further by Harvey and Ftaclas ${ }^{27}$ along with the computer analysis program FRED to show that the image plane diffraction patterns from curved secondary support structure is less than $10^{-6}$ where-as the image plane diffraction patterns from straight line secondary support structure are $\sim 10^{-2}$.

Based on our intuitive understanding of diffraction from curved segments we designed a pupil topology for a "first look" at the diffraction effects. The design we chose is shown in Figure 8 below. We selected a 10-meter Cassegrain primary with an obscuration ratio of 0.16 and six curved secondary mirror support struts, each with a $30^{\circ}$ arc of a circle and $20 \mathrm{~mm}$ wide gaps. There are three rings or zones of segments curved on all sides. Each zone contains 12 curved sided segments to create a telescope entrance pupil that has 36 segments. This topology is shown in Figure 7, below.

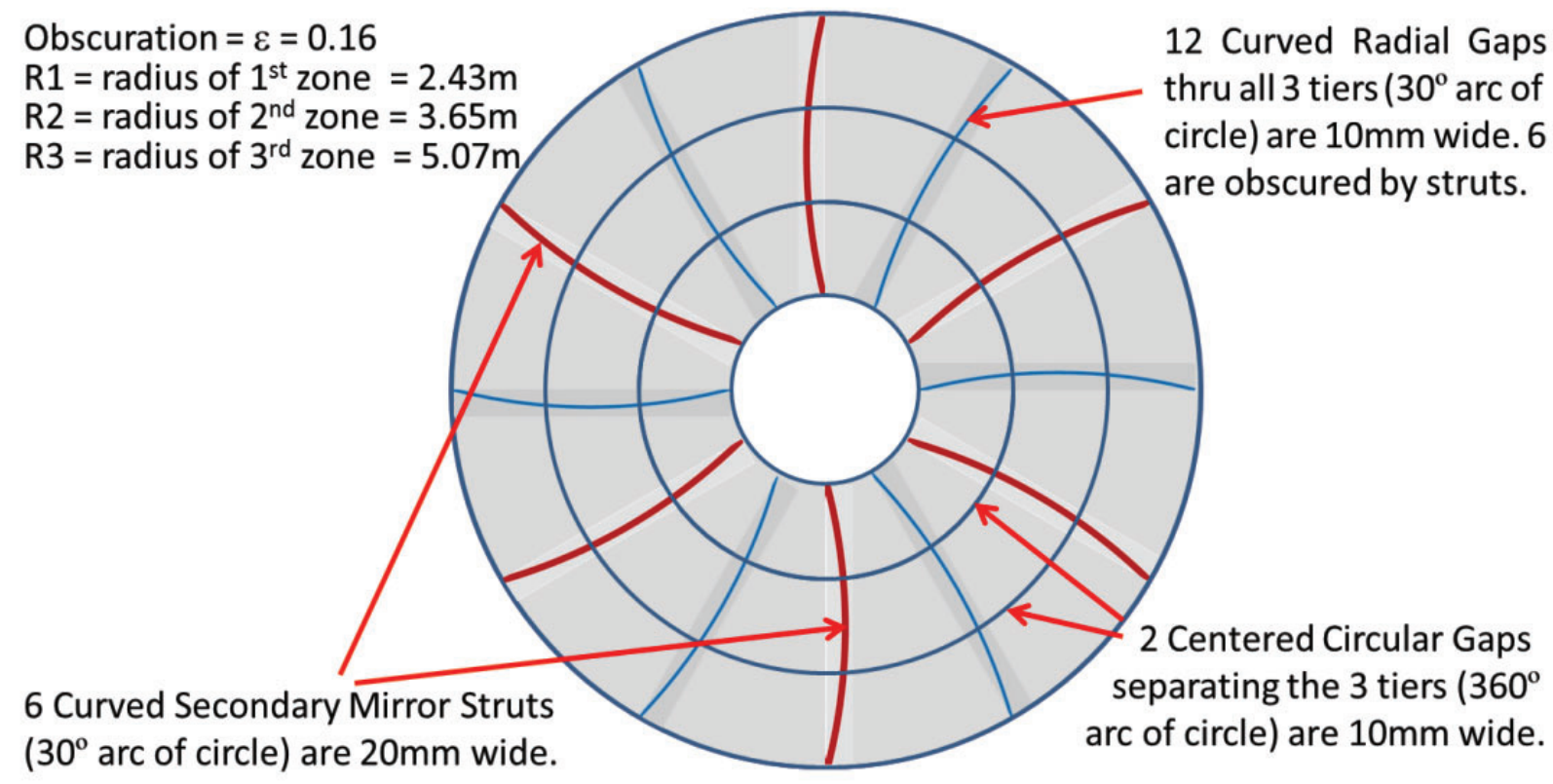

Figure 7 Cassegrain primary with an obscuration ratio of 0.16 and six curved secondary mirror support struts (shown in Red), each with a $30^{\circ}$ arc of a circle and $20 \mathrm{~mm}$ wide. These secondary support struts cast shadows on the primary mirror. Since the optical system stop is at the primary mirror, these shadows will move across the aperture as the image plane field point is changed. There are three rings or zones of mirror segments curved on all sides. Each zone contains 12 curved sided segments to create a telescope entrance pupil that has 36 segments. 
Figure 8, below shows how
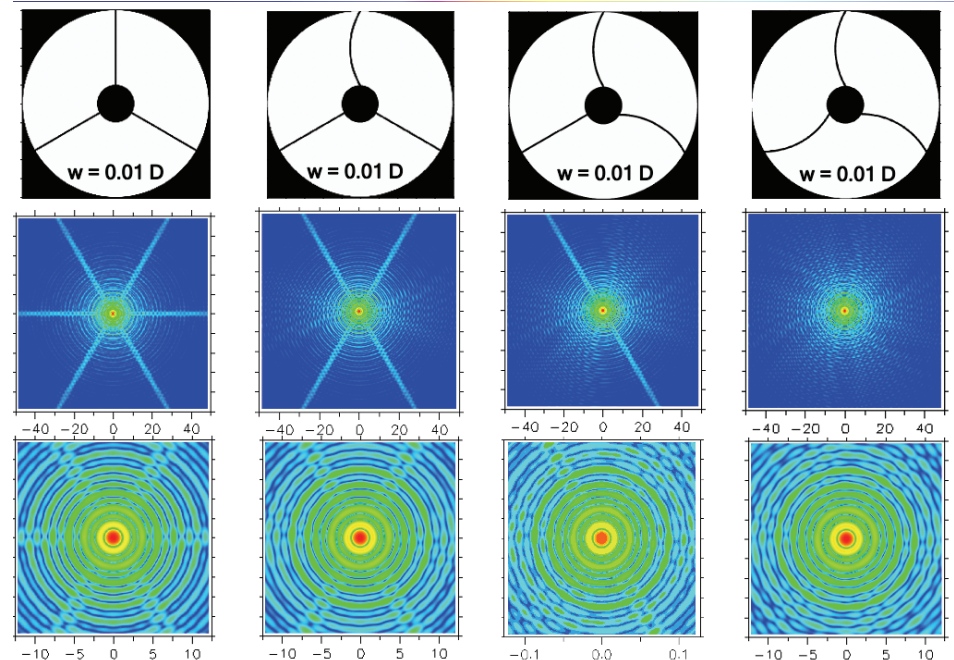

Figure 8 Simulated diffraction effects for an annular aperture ( $=0.16)$ and three straight spiders $(w=0.01 D)$ as each spider is sequentially converted to an arc of a circle.

Figure 8 shows the dramatic change in diffracted light at the image plane as we curve the secondary support system.

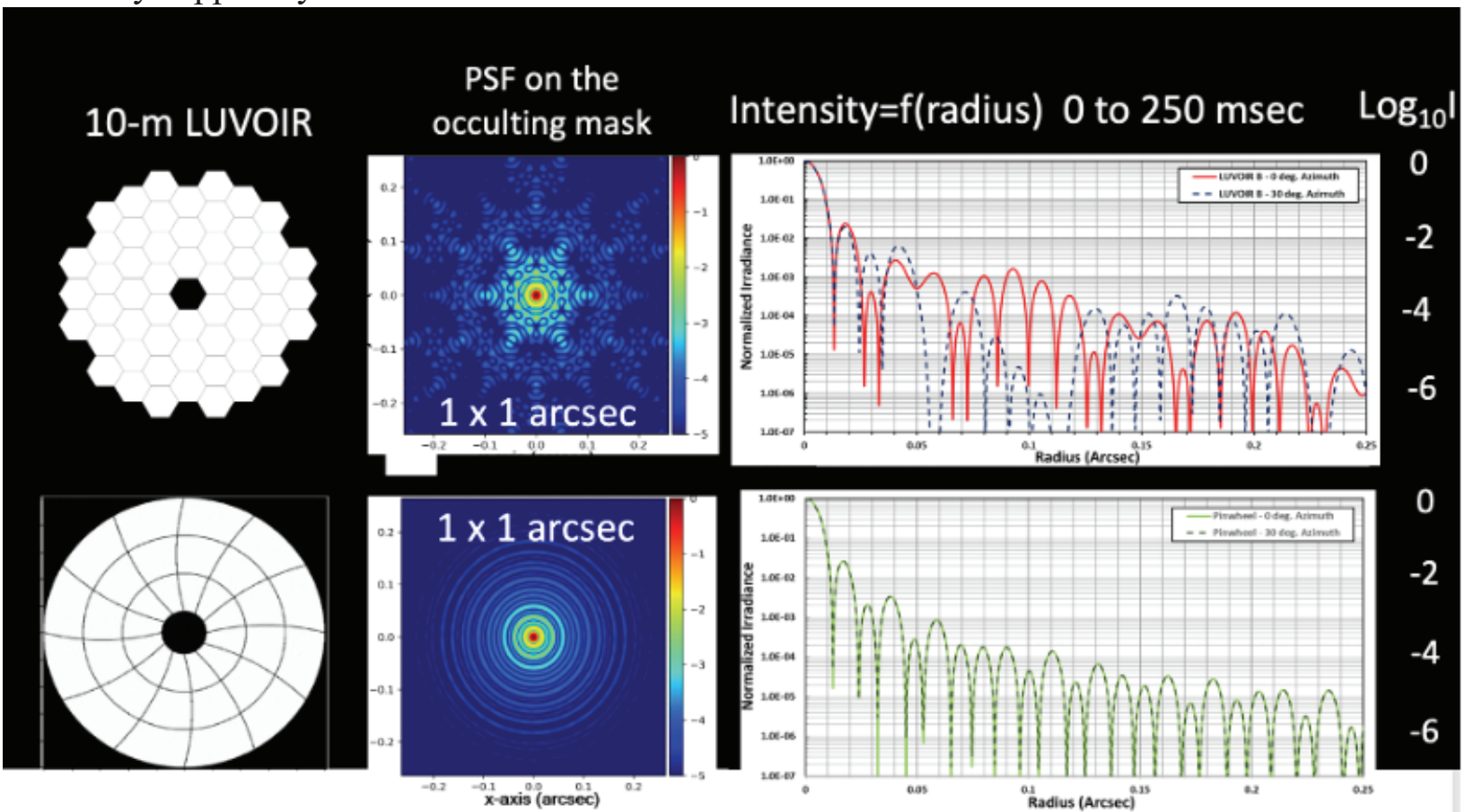

Figure 9 Image plane linear-scale monochromatic intensity distribution from three 10-m diameter pupil topologies: unobstructed, spider and pinwheel are shown at field radius 0.75 arc-second for azimuth angles -180 to +180 degrees. Monochromatic diffraction patterns for a modified LUVOIR-B aperture stretched from 8-m to 10-m diameter with 5-mm segment gaps is compared to a 10-m diameter pinwheel aperture with 5-mm segment gaps. Top row $L$ to $R$ : modified LUVOIR-B aperture map, $\log _{10}$ stretch of the PSF and a plot of the normalized irradiance as a function of radius for $0^{0}$ (red) and $30^{\circ}$ (blue dots) azimuth angle. Bottom row $L$ to $R$ : pinwheel aperture 
map, $\log _{10}$ stretch of the PSF and a plot of the normalized irradiance as a function of radius for $0^{\circ}$ (green) and $30^{\circ}$ (grey dots) azimuth angle. The center plots on a blue background show the expected system point spread function that falls onto the coronagraph occulting mask. The PSF structure shown at top-center indicates that many false-positive identification of exoplanets may occur and exoplanets can be masked if a hex segmented aperture is used.

Figure 9 shows a plot of monochromatic intensity, on a linear scale, as a function of azimuth angle at field 0.75 arc sec. for three 10-m diameter pupil architectures: unobstructed, spider and pinwheel. The computation was performed using MatLab. Computational capacity limited the size of the sample interval across the pupil and we believe that may have resulted in an incorrect representation of the image plane diffracted light. However, several features in Fig 9 are worth noting. The profile for the 4-spider mask, shown in red, is much higher than that for the pinwheel which indicates that the pinwheel pupil is making a contribution to smoothing out the diffraction pattern. The prominent dip in energy at the feet of the spider diffraction pattern is probably the result of the very narrow bandwidth of this monochromatic computation. The noise on the pinwheel is probably caused by having an insufficient number of samples across the pupil, which was dictated by the array sizes and the computational time limits of Matlab.

Consequently, we decided to drop MatLab as our computational tool and turn to Photon Engineering. LLC and the FRED software. Computations using FRED were successful and are shown in the paper: SPIE Proc 10698-6027.

Are curved sided segments purely in the imagination of the theorist, or can one fabricate curved sided segments to optical tolerances? If yes, then we can create a large aperture in space for a deployable full-aperture diffraction limited space telescope. Ten or even twenty meter clear aperture image quality will be possible. In the next section, we suggest physical processes and techniques to enable very large aperture space telescopes for the next generation.

\section{MANUFACTURE OF CURVED-SIDED SEGMENTS}

Figure 10 illustrates the typical shape of mirror segments forming the spiral aperture. This is fundamentally the same situation as that for making hexagonal segments.

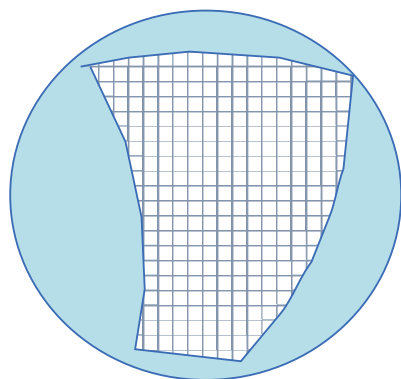

Figure 10: Pinwheel pupil segment is shown within its ZERODUR ${ }^{\circledR}$ roundel. The rectangular grid support structure is shown to indicate an isogrid back structure. Engineering details of this isogrid structure would follow detailed structural engineering for thermal, mechanical, and structural design of the mirror as a spaceflight element attached to a back-plane.

Manufacture of curved-sided non-roundel aspheric-surface segments is fundamentally similar to the manufacture of hexagonal-sided aspheric-surface segments, provided the radius of curvature of the sides are gentle, like those shown in Figure 10. The primary mirrors of both the Thirty Meter Telescope (TMT, 30-m) and the European Extremely Large Telescope (ELT, 39- 
$\mathrm{m}$ ), as were the Keck Observatory and other mirrors, are fabricated by polishing as a roundel, then the edges parted to form the hexagonal end form.

In the case of the European Extremely Large Telescope (ELT) 931 curved-face hexagonal shaped segments are being made. Each is sized to $1.45-\mathrm{m}$ point-to-point and the mirror material is ZERODUR ${ }^{\circledR}$, a heritage glass-ceramic developed by SCHOTT for astronomical telescopes. This material exhibits extreme stability as temperature changes. In the case of the ELT, REOSC corporation then machines the hexagonal perimeter shape of the nearly finished roundel, and minute changes in optical figure are corrected using small-tool ion beam figuring (Figure 11). REOSC corporation is delivering mirror segments at the rate of approximately one per day, so it is expected that the time for correction is only a day or two. We expect the process and results would be similar for the spiral form we address.

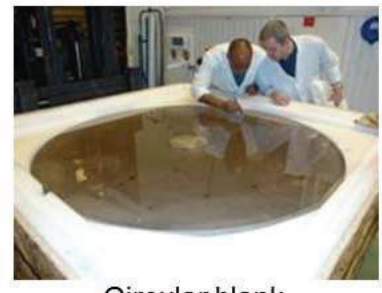

Circular blank

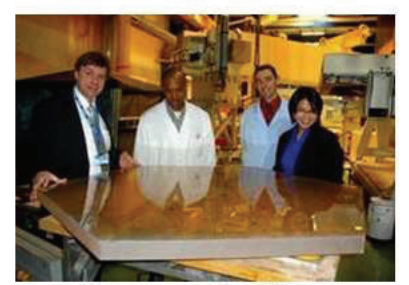

Hexagonal cutting

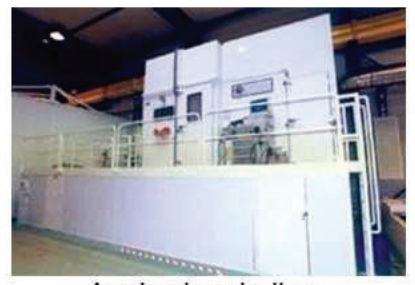

Aspheric grinding

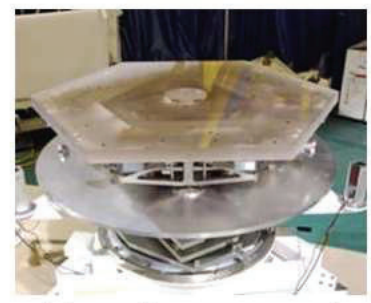

Integration on support

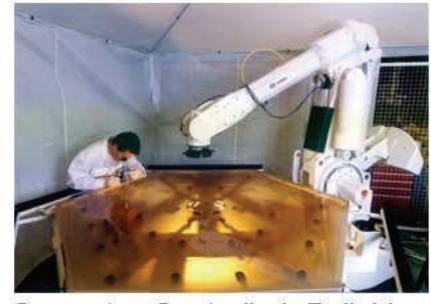

Computer Controlled Polishing

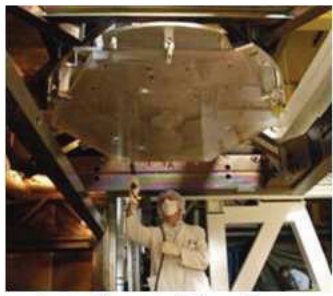

Ion Beam Figuring

Figure 11: REOSC corporation production plan for ELT hexagonal segments (optro-2018-02-07_first_steps_in_ELT_optics_polishing_mnscpt.pdf)

While the parted surface would approximate a continuous surface with only small corrections due to slight change in the internal stress field, subsequent deterministic small tool polishing, even with a tool as small as a centimeter in diameter, the very edge of the form, whether hex or spiral shaped, cannot be reached. Thus, realistic evaluation of any form must take into account a clear aperture allowance for what is termed "edge roll-off". In the case of the JWST mirror segments Figure 12, where entire optical processing is as a hexagon, the average allowance for edge roll-off was $\sim 5$-mm average around the perimeter, with the hex included angle equaling 120 degrees. With the pinwheel form, the included angle may even be acute, and this is one challenge to be faced. Subsequent analysis will consider the effects of clear aperture error allocation, both at the corners and along the continuous edges. 


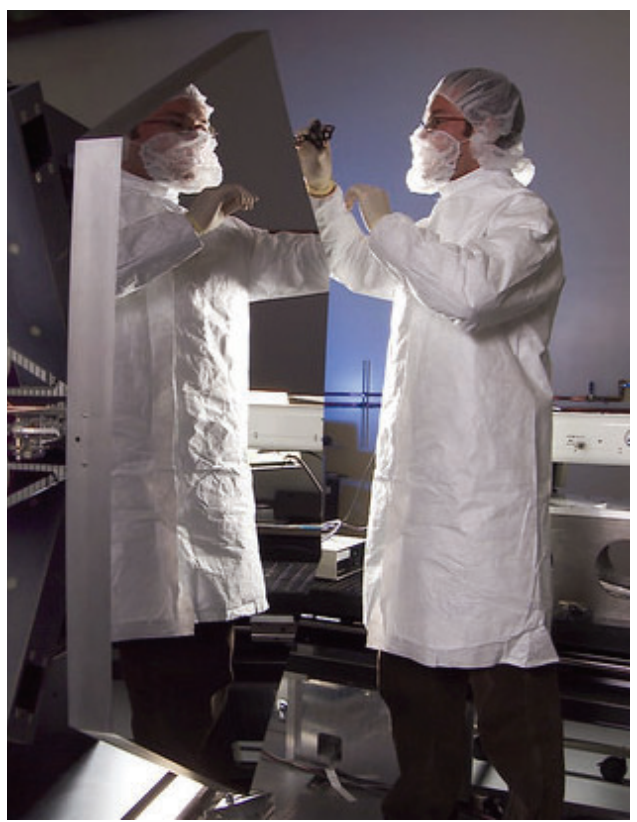

Figure 12: A JWST hexagonal segment is shown during optical finishing at L-3 Tinsley. An average clear-aperture allowance around the edge of $5 \mathrm{~mm}$ was achieved while polishing the lightweight segment from the start in hexagonal form.

(NASA Photograph...

https://jwst.nasa.gov/content/observatory/ote/mirrors/index.html)

Various approaches to optical finishing and parting into shape are possible, and the optimum approach may depend on the standard methods of the entity selected for optical polishing.

a) As illustrated by REOSC corporation for the ELT, standard polishing as a roundel and edges parted, then deterministic small tool correction for any minute shape change

○ Option: before polishing, undercut the desired shape to within TBD millimeters of the mirror surface, stabilize the assembly, and part after polishing. It is presumed that this would approximate the stress relaxation state of the mirror when cut to shape. However, this imposes requirements on how each part of the mirror is held during polishing, transport and test operations.

b) Stress mirror polishing as a roundel and edges parted, then deterministic small tool correction for any minute shape change [A]

c) First cut the mirror shape, "wax" the removed segments back in place to return to a roundel, and standard polish as a roundel.

Approaches (a) and (b) have been the most typical for segmenting to date, though these have been for solid roundels, not light-weighted. Method (c), while it would minimize allocation for edge roll-off, imposes significant additional challenges and risks on the optical processing. In all cases, from the perspective of segment damage, a small bevel is needed to blunt the cut edges.

A large spaceborne telescope like the NASA studied LUVOIR will require significant mass reduction and high eigenfrequencies for each segment. Thus, light-weighting is requisite. In the case of ZERODUR ${ }^{\circledR}$, typically an open-back isogrid pattern is cut, with thin ribs, and facesheet-to-lightweighting cell size being determined by the optical finishing practice being 
used. Other materials like ULE may evoke different lightweighting approaches. However, this introduces additional approaches:

d) Light-weighting is first produced. Then method (a), (b) or (c) - above.

e) Light-weighting is machined into the optically finished roundel by method

(a) or (b). Small tool corrections are applied to correct any changes from light-weighting, and from cutting to shape. This is the method applied to the Webb primary mirror segments, largely due to the segments being made of beryllium, where special considerations apply.

The technologies to be demonstrated are 1) the degree of clear aperture allowance needed, both along the walls, and especially at the discontinuous points, and variants to the methods used on ground telescopes, and 2) the complexity imposed by lightweighting. In all cases, we believe there are viable solutions to the spiral form equivalent to established methods for hexagons.

References for mirror fabrication technology fundamental to creating curved sided segments are found in the literature ${ }^{28}, 29$

\section{REFERENCES}

${ }^{1}$ Joseph W. Goodman (2005) "Fourier Optics" Roberts and Company book ISBN 9747077-2-4

2 J. D. Gaskill (1978) "Linear Systems, Fourier Transforms and Optics”, John Wiley \& Sons, NY 400 pages.

${ }^{3}$ Breckinridge, J. B. , R. A. Chipman, M. Kupinski, J. E. Harvey, J. Davis and B. Daugherty (2020) "Threshold Raw Retrieved Contrast in Coronagraphs Is Limited by Internal Polarization:, NASA Final Report for Grant \# NNX17AB29G, Jan 15, 2020 - 131 page report. Download from: https:/exoplanets.nasa.gov/exep/technology/TDEM-awards/

${ }^{4}$ Breckinridge, J. B., J. E. Harvey, R. Irvine, R. Chipman, M. Kupinski, J. Davis, D-W Kim, D. S. Ewen, C. F. Lillie and T. Hull (2019) "ExoPlanet Optics: conceptual design processes for stealth telescopes", Proc SPIE 11115-17

${ }^{5}$ Clark, N. and Breckinridge, J. B. (2011) "Polarization compensation of Fresnel aberrations in telescopes" SPIE Proc. 8146 \# 814600

${ }^{6}$ Polidan, R., J. B. Breckinridge, C, F, Lillie, H. A. MacEwen, M. R. Flannery and D. R. Dailey (2016) "Innovative telescope architectures for future large space observatories", J. Astron.

Telesc. Instrum. Vol 2

${ }^{7}$ Harvey, J. E. ; R. G. Irvin, K. Crabtree, R. N. Pfisterer and J. B. Breckinridge (2018);

"Diffraction Analysis of Large Segmented Mirror Concepts for Exoplanet Exploration" SPIE

Proc. 10698-60

${ }^{8}$ Trunbull, M. C., ExoCat-1: The Nearby Stellar Systems Catalog for Exoplanet Imaging

Missions, arXiv:1510.01731 [astroph.SR] arXiv:1510.01731 [astro-ph.SR] 2015.

${ }_{9}^{9}$ Beckwith, S. V. W.; M. Stailelli, A. M. Koekemoer, J. A. R. Calswell, et. al.; "The Hubble Ultra Deep Field" Astron. J. 132:1729-1755 (2006).

${ }^{10}$ Breckinridge, J. B., T. G. Kuper and R. V. Shack "Low-scattered light camera: a model" Optical Engineering 23, pp 816-820; (1984)

${ }^{11}$ Pfister, R. N. , Harvey, J. E. and J. B. Breckinridge, "The role of narrow angle forward surface scatter and particulate scatter in exoplanet exploration", Proc SPIE 10698-188, 2018.

12 Breckinridge, J. B. , W. S. T. Lam and R. A. Chipman (2015) "Polarization Aberrations in Astronomical Telescopes: The Point Spread Function”, Publications of the Astronomical Society of the Pacific (PASP), 127:445-468 May 
${ }^{13}$ Breckinridge, J. B. “The pinwheel pupil discovery: exoplanet science and improved processing with segmented telescopes" Paper \# 439.04 231st meeting of the American Astronomical Society, Washington, DC. 2018.

${ }^{14}$ Harvey, J. E. , R. G. Irvin, K. Crabtree, R. N. Pfister and J. B. Breckinridge "Diffraction Analysis of Large Segmented Mirror Concepts for Exoplanet Exploration", SPIE proceedings 10698-060, 2018.

${ }^{15}$ J. B. Breckinridge, J. E. Harvey, K. Krabtree \& T. Hull (2018) "ExoPlanet Telescope diffracted light minimized: The pinwheel pupil solution" Proc. SPIE 10698-1P

${ }_{16}$ Pueyo, L., and Norman, C., (2013) High Contrast Imaging with an arbitrary aperture: active compensation of aperture discontinuities. The Astroph. Journ., 769:102 (31pp),

doi:10.1088/0004-637X/769/2/102 C

${ }^{17}$ Garreth Ruane, Dimitri Mawet, Jeffrey Jewell, Stuart Shaklan, "Performance and sensitivity of vortex coronagraphs on segmented space telescopes," Proc. SPIE 10400, 104000J ; doi: $10.1117 / 12.2274508$ (2017)

18 Jewell, J. G. Ruane, S. Shaklan, D. Mawet, D. Redding, "Optimization of coronagraph design for segmented aperture telescopes," Proc. SPIE 10400, 104000H; doi: 10.1117/12.2274574 (2017)

19 Vanderbei, R.J., Spergel, D.N., and Kasdin, J.N., "Spiderweb Masks for High Contrast Imaging,: ApJ vol. 590-593, 2003.

${ }^{20}$ Lillie, C., M. Flannery, D. Dailey "Segmented Telescopes For Coronagraphs" Proc of SPIE Vol. 5487 0277-786X/04/\$15 - doi: 10.1117/12.552348 (2004)

${ }^{21}$ Alexis Carlottia,b, Dimitri Mawetc, Laurent Pueyo Optimal apodizers for the vector vortex coronagraph with on-axis telescopes, Proc. of SPIE Vol. 8864, 88641P; CCC code: 0277786X/13/\$18 - doi: 10.1117/12.2024511

${ }^{22}$ Breckinridge, J. B. "The pinwheel pupil discovery: exoplanet science and improved processing with segmented telescopes" Paper \# 439.04 231st meeting of the American Astronomical Society, Washington, DC. 2018.

${ }^{23}$ C. H. Werenskiold (1941) "Improved Telescope Spider Design", Journ. Royal Astron. Soc. Canada, 35, 268-272. AND C. H. Werenskiold, "A note on curved spiders,' Sky Telesc. 262263, 1969

${ }^{24}$ Richter, J. L. (1984) "Spider diffraction: a comparison of curved and straight legs", AO, 23 1907-1913

${ }^{25}$ Harvey, J. E. , R. G. Irvin, K. Crabtree, R. N. Pfister and J. B. Breckinridge "Diffraction Analysis of Large Segmented Mirror Concepts for Exoplanet Exploration”, SPIE proceedings 10698-060, 2018.

${ }^{26}$ J. E. Harvey, R. G. Irvin and R. N. Pfisterer, "Modeling Physical Optics Phenomena by Complex Ray-tracing”, Opt. Eng., 54 (3), 035105 (2015). doi: 10.1117/1.OE.54.3.035105.

${ }^{27}$ Harvey, J. E. and C. Ftaclas, "Diffraction Effects of Telescope Secondary Mirror Spiders upon Various Image Quality Criteria”, Appl. Opt. 34, 6337-6349 (1995).

${ }^{28}$ Jedamzik, R., C. Kunisch, T. Westerhoff, Muller, U., Daniel, J. “ZERODUR for stress mirror polishing II: Improved modeling of the material behavior." Proc. SPIE. 8450 (2012)

${ }^{29}$ Ralf Jedamzik, Clemens Kunisch, Thomas Westerhoff, Ulrich Müller, Jay Daniel, ZERODUR for stressed mirror polishing II: improved modeling of the material behavior, Proc. SPIE. 8450, (2012) Modern Technologies in Space- and Ground-based Telescopes and Instrumentation II 